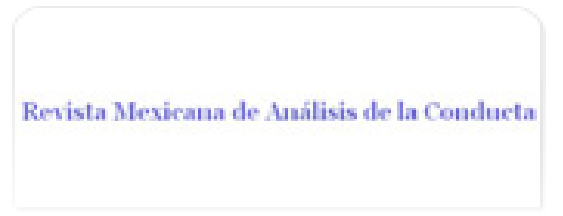

Revista Mexicana de Análisis de la Conducta ISSN: 0185-4534

editora@rmac-mx.org

Sociedad Mexicana de Análisis de la Conducta México

SÁNCHEZ-CARRASCO, LIVIA; NIETO, JAVIER

RECUPERACIÓN DE RESPUESTAS: UNA REVISIÓN DE LA EVIDENCIA Y DEL MODELO DE RECUPERACIÓN DE INFORMACIÓN

Revista Mexicana de Análisis de la Conducta, vol. 35, septiembre, 2009, pp. 45-59

Sociedad Mexicana de Análisis de la Conducta

Guadalajara, México

Disponible en: http://www.redalyc.org/articulo.oa?id=59312304005

- Cómo citar el artículo

- Número completo

- Más información del artículo

Página de la revista en redalyc.org

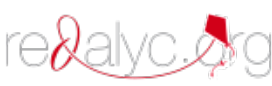

Sistema de Información Científica

Red de Revistas Científicas de América Latina, el Caribe, España y Portugal Proyecto académico sin fines de lucro, desarrollado bajo la iniciativa de acceso abierto 


\title{
RECUPERACIÓN DE RESPUESTAS: UNA REVISIÓN DE LA EVIDENCIA Y DEL MODELO DE RECUPERACIÓN DE INFORMACIÓN
}

\author{
RETRIEVAL OF RESPONDING: A REVIEW OF DATA AND THE \\ INFORMATION RETRIEVAL MODEL
}

\author{
LIVIA SÁNCHEZ-CARRASCO Y JAVIER NIETO' \\ UNIVERSIDAD NACIONAL AUTÓNOMA DE MÉXICO \\ FACULTAD DE PSICOLOGÍA
}

\begin{abstract}
RESUMEN
Los fenómenos de recuperación de respuestas (i.e. renovación contextual, restablecimiento y recuperación espontánea) sugieren que, a pesar del decremento en la frecuencia de la respuesta, las asociaciones establecidas en la adquisición se conservan intactas durante la extinción. En el presente trabajo se hace una revisión de la evidencia sobre estos fenómenos, así como del modelo de recuperación de información de Bouton, el cual propone que el contexto funciona como un configurador de ocasión. Posteriormente, se discuten los problemas del modelo de interferencia y se describe la teoría atencional del procesamiento contextual, que incorpora a la atención como un mecanismo que favorece la dependencia contextual de la información. Finalmente, se presentan algunas consideraciones que deben incorporarse en el desarrollo de estos modelos y en la investigación en el área.

Palabras clave: renovación, restablecimiento, recuperación espontánea, y señales contextuales.

1. Este trabajo fue financiado por los proyectos: PAPIIT307509 otorgado por la DGAPA-UNAM y el proyecto Conacyt 83319. La correspondencia sobre este artículo debe dirigirse a Javier Nieto, Facultad de Psicología, División de Estudios de Posgrado, Universidad Nacional Autónoma de México, Cubículo 16, Edificio D, 1er Piso. C. P. 04510, o al correo electrónico: janigu@unam.mx.

Recibido: 11 de abril de 2009. Revisado: 8 de junio de 2009. Aceptado: 17 de julio de 2009.
\end{abstract}




\begin{abstract}
Response retrieval phenomena (i.e., context renewal, reinstatement and spontaneous recovery) suggest that despite reduction in responding, the associations established during acquisition are left intact by extinction. This paper presents a review of evidence related to these phenomena, and introduces Bouton's Information Retrieval Model that proposes that the context may be seen as an occasion setter. Then, some shortcomings of the information retrieval model are discussed, and the attentional theory of context processing is presented, this theory proposes that attention to contextual cues promotes context dependency of information. Finally, we present some considerations that must be incorporated into these models and in the ongoing research in the area.
\end{abstract}

Keywords: renewal, reinstatement, spontaneous recovery, and contextual cues.

Los procedimientos de condicionamiento utilizados en el estudio de la conducta son: el condicionamiento clásico o pavloviano y el condicionamiento instrumental u operante. En el primero se empareja un estímulo condicional (EC) con un estímulo incondicional (EI) y en el segundo, la emisión de una respuesta (R) es seguida por una consecuencia (C). Los estudiosos de la conducta han utilizado estos procedimientos para analizar las variables independientes de las cuales resulta el comportamiento, así como las relaciones funcionales existentes entre éste y los eventos ambientales (Skinner, 1950). Por otro lado, los teóricos del aprendizaje asociativo emplean los mismos procedimientos para determinar los mecanismos cognitivos que subyacen al cambio conductual (Dickinson, 1980; Hall, 2002).

Desde la perspectiva del aprendizaje asociativo, los cambios conductuales observados durante el condicionamiento se consideran resultado de cambios en las conexiones neuronales o en la actividad del sistema nervioso, que algunos autores denominan sistema nervioso conceptual (Hall, 2002). Este sistema está compuesto de unidades, nodos o representaciones que establecen asociaciones entre sí y su fuerza modula la expresión del comportamiento. En consecuencia, las investigaciones desarrolladas en esta área emplean los procedimientos de condicionamiento para analizar los mecanismos que subyacen al aprendizaje. Así, por ejemplo, después de emparejar repetidamente un EC con un El, se observa un incremento en la probabilidad de ocurrencia de la respuesta condicional $(\mathrm{RC})$ ante la sola presentación del EC. De igual forma, cuando una respuesta instrumental es seguida por un reforzador, su probabilidad de ocurrencia aumenta. Por otro lado, si las con- 
diciones experimentales se mantienen constantes y se omite la presentación del El o el reforzador, se observa un decremento en la probabilidad de ocurrencia de la respuesta, que se conoce como extinción. Desde los trabajos pioneros de Pavlov (1927) y Konorski (1948), los teóricos del aprendizaje han intentado determinar los mecanismos asociativos que subyacen a diversos fenómenos de aprendizaje como: adquisición, extinción, inhibición latente, bloqueo, entre otros. En particular, los estudios sobre la extinción pretenden determinar los mecanismos asociativos o no asociativos responsables de la reducción en la respuesta (Rescorla, 2001).

En la primera parte del presente trabajo se analiza la evidencia relacionada con los mecanismos responsables de la reducción en la respuesta observada durante extinción, la cual sugiere que la extinción no destruye las asociaciones establecidas en la adquisición. Posteriormente, se examinan diversos fenómenos que se encuentran relacionados con el estudio de la extinción, los cuales sugieren que la ejecución de las respuestas extinguidas es dependiente de contexto. Finalmente, se discuten algunos de los modelos que describen los mecanismos responsables de la extinción y la dependencia contextual, así como sus principales limitaciones.

\section{EXTINCIÓN}

Actualmente existen al menos dos grupos de teorías que pretenden describir los mecanismos causantes de la extinción. El primer grupo asume que el decremento en la respuesta resulta de la ruptura o disminución en la fuerza de las asociaciones EC-El o R-C establecidas en el condicionamiento (e.g. Estes, 1950; Rescorla \& Wagner, 1972). El segundo grupo de teorías supone que las asociaciones establecidas durante el condicionamiento prevalecen en la extinción; sin embargo, éstas compiten con las asociaciones de tipo inhibitorio establecidas en dicha fase (e.g. Konorski, 1948; Pavlov, 1927; Rescorla, 1979). Actualmente existen dos clases de evidencia que sustentan a este último grupo de teorías. La primera proviene de experimentos en los que se evalúa, a través de procedimientos de devaluación o transferencia, el estado de las asociaciones EC-El o R-C extinguidas (Colwill \& Rescorla, 1988; Rescorla, 1992; 1993; 1996b; 1996a).

En los procedimientos de devaluación la emisión de dos respuestas instrumentales es seguida de dos consecuencias diferentes (pellets o sucrosa líquida). Posteriormente, se devalúa el valor motivacional de alguna de las consecuencias emparejándolas con cloruro de litio. Como resultado de esta devaluación se observa un decremento en la respuesta asociada con la consecuencia devaluada, este resultado sugiere que los organismos han aprendido asociaciones R-C durante el condicionamiento. Adicionalmente, se ha 
demostrado que el efecto de la devaluación de la consecuencia se observa aún después de extinguir la respuesta instrumental (Rescorla, 1993) o una RC (Rescorla, 1996a). Por tanto, se ha sugerido que las asociaciones R-C o EC-El prevalecen después de la extinción.

Los estudios que emplean procedimientos de transferencia han permitido analizar la posibilidad de que asociaciones entre el estímulo y la omisión de la consecuencia esperada (estímulo-no consecuencia) afecten la motivación para responder, aun cuando las asociaciones R-C estén vigentes (Colwill \& Rescorla, 1988). Por ejemplo, Rescorla (1992) entrenó a ratas a emitir dos respuestas instrumentales, la emisión de una de ellas se reforzó con sucrosa líquida, mientras la otra se reforzó con pellets. Posteriormente, se entrenó la emisión de una tercera respuesta, la cual fue reforzada con pellets o sucrosa líquida, dependiendo de si estaba presente una luz o un tono. En la siguiente fase, se extinguió la emisión de la tercera respuesta en presencia de alguno de estos estímulos; y finalmente, se condujo una sesión de prueba en la que los sujetos podían emitir cualquiera de las respuestas originales cuando se presentaba la luz o el tono. A pesar de la extinción, los resultados mostraron que la presentación de cualquiera de los estímulos producía un incremento selectivo en la respuesta instrumental con la que compartía la consecuencia. Por tanto, la extinción tampoco afecta las asociaciones estímulo-consecuencia.

En conclusión, los resultados de estos experimentos sugieren que las asociaciones R-C y E-C se preservan después de la extinción, por tanto la ruptura de estas asociaciones o el establecimiento de asociaciones E-no $\mathrm{C}$ parecen no ser los mecanismos responsables del decremento en la respuesta observado en extinción. Evidencia reciente indica que los procedimientos de extinción resultan en el establecimiento de asociaciones inhibitorias entre los estímulos discriminativos y la respuesta que los sujetos emiten durante la extinción (Colwill, 1993). Sin embargo, la evidencia obtenida en estos estudios es indirecta, por lo que aún se desconoce el tipo de asociaciones que se establecen durante la extinción (Gámez \& Rosas, 2005).

La segunda línea de evidencia que sustenta la idea de que las asociaciones establecidas durante el condicionamiento no se destruyen totalmente en la extinción, proviene de estudios que muestran la recuperación de respuestas extinguidas a través de fenómenos como la recuperación espontánea, la renovación contextual y el restablecimiento. El estudio de estos fenómenos ha permitido analizar los mecanismos que subyacen a la inhibición de la respuesta durante la extinción, así como el proceso responsable de su recuperación posterior. 


\section{RENOVACIÓN CONTEXTUAL}

La renovación contextual ha sido ampliamente estudiada por Bouton y colaboradores (Bouton \& Bolles, 1979a; Bouton \& Swartzentruber, 1986), y se observa cuando se expone a los sujetos a un contexto de estímulos distinto del empleado en la fase de extinción. En los experimentos con animales no humanos, los cambios en el contexto se hacen modificando la cámara experimental ya sea pintando de diferentes colores las paredes, añadiendo aromas distintivos, alterando las dimensiones de la caja o bien aumentando el diámetro de las rejillas que constituyen el piso. Así, si se entrena la emisión de una respuesta en un contexto $A$, se extingue en un contexto $B$ y finalmente se conduce, en el contexto $A$, una prueba en extinción, se observa un incremento de la frecuencia de la respuesta extinguida, que se conoce como renovación contextual $A B A$. La recuperación de respuestas (i.e. renovación $A B C$ ) se ha observado también cuando las tres fases del experimento se conducen en diferentes contextos (Bouton \& Swartzentruber, 1986), así como cuando la adquisición y la extinción se realizan en el mismo contexto, mientras la prueba se conduce en un contexto nuevo (i.e. renovación $A A B$ ) (Bouton \& Ricker, 1994).

La renovación contextual ha mostrado ser un hallazgo generalizable a diferentes procedimientos de condicionamiento y se ha reportado empleando procedimientos de supresión condicionada (Bouton \& Bolles, 1979a), condicionamiento clásico apetitivo (Bouton \& Peck, 1989), condicionamiento instrumental (Nakajima, Tanaka, Urushihara \& Imada, 2000), condicionamiento de aversión a sabores (Rosas \& Bouton, 1997) y en tareas de juicios causales con humanos (Paredes-Olay \& Rosas, 1999). Además, se ha encontrado renovación cuando el contexto de extinción lo proporcionan estímulos interoceptivos como drogas (Bouton, Kenney \& Rosengard, 1990) o estrés (Ahlers \& Richardson, 1985).

\section{RESTABLECIMIENTO}

El restablecimiento, o reinstauración, es otro procedimiento que permite observar la reaparición de respuestas extinguidas y consta de tres fases. En la primera fase, se entrena una RC o instrumental, en la segunda fase se extingue la respuesta previamente condicionada y en la última fase los sujetos son expuestos en forma no contingente al El o reforzador empleado durante la primera fase. La prueba de restablecimiento consiste en evaluar la RC ante el EC, o la frecuencia de emisión de la respuesta instrumental ante el manipulandum. Esto se hace de dos maneras: en una se reexpone al sujeto al El o reforzador en la misma sesión (Doughty, Reed \& Lattal, 2004; Franks 
\& Lattal, 1976; Reid, 1958; Rescorla \& Skucy, 1969) y en la otra se expone al sujeto al EC o al manipulandum $24 \mathrm{~h}$ después de la reexposición al El o al reforzador (Baker, Steinwald \& Bouton, 1991; Bouton \& Bolles, 1979b; Rescorla \& Cunningham, 1977; Rescorla \& Heth, 1975). En los dos casos, cuando la sesión de prueba se compara con la sesión de extinción, los resultados muestran un incremento en la respuesta. Aunque es posible suponer que la realización simultánea de la prueba de restablecimiento y la reexposición al El o reforzador resulta en el incremento en la respuesta por el reforzamiento accidental, no existe evidencia que indique que, en este procedimiento, el restablecimiento sea diferente al que se observa cuando la prueba se realiza $24 \mathrm{~h}$ después de reexponer a los sujetos al El o reforzador.

La evidencia sobre restablecimiento de respuestas ha mostrado que no existe una relación sistemática entre el número de sesiones de extinción y la frecuencia de emisión de la respuesta durante la fase de prueba (Franks \& Lattal, 1976; Rescorla \& Skucy, 1969). También se ha mostrado que el restablecimiento de la respuesta es una función creciente del número de sesiones de condicionamiento (Uhl, 1973) y de la tasa de respuestas registrada en la última sesión de condicionamiento (Doughty et al., 2004; Franks \& Lattal, 1976). Finalmente, existe evidencia de que el restablecimiento de una respuesta es selectivo de la consecuencia empleada para su condicionamiento (Delamater, 1997; Ostlund \& Balleine, 2007).

\section{RECUPERACIÓN ESPONTÁNEA}

Pavlov (1927) reportó un experimento en el que durante varios ensayos emparejó un EC (sonido de un metrónomo) con un El (comida) hasta que se estableció la RC de salivación. Posteriormente, implementó un procedimiento de extinción en el cual presentó el EC, sin el El, hasta que la respuesta de salivación se redujo a niveles cercanos a cero ( $0 \mathrm{ml}$ de saliva). Después de transcurrido un periodo de descanso de $2 \mathrm{~h}$ reexpuso a los sujetos al EC y observó un incremento en la RC de salivación al que denominó recuperación espontánea.

En un estudio posterior, Ellson (1938) mostró la recuperación espontánea de respuestas instrumentales; para ello entrenó a un grupo de ratas a presionar una palanca para obtener alimento, $24 \mathrm{~h}$ después las sometió a un procedimiento de extinción hasta que no se registraron respuestas a la palanca durante 5 min consecutivos. Posteriormente, introdujo un periodo de descanso que varió de 5 min a $3 \mathrm{~h}$, durante el cual mantuvo a los sujetos en su jaula habitación. Finalmente, regresó a los sujetos a las cámaras de condicionamiento donde continuó la extinción por una sesión y observó un incremento (i.e. recuperación espontánea) en la tasa de respuestas a la 
palanca, que fue mayor para los sujetos que descansaron $3 \mathrm{~h}$ que para los que descansaron $5 \mathrm{~min}$. Este hallazgo es consistente con la evidencia reportada en diversos estudios que muestran mayor recuperación de la respuesta extinguida conforme aumenta la duración del periodo de descanso (Ellson, 1938; Robbins, 1990).

\section{INHIBICIÓN CONDICIONADA}

Como se mencionó anteriormente, se ha sugerido que durante la extinción se establecen asociaciones inhibitorias (Konorski, 1948; Pavlov, 1927), por lo que es posible sugerir la existencia de una relación entre el condicionamiento inhibitorio y la extinción. Bouton y Nelson (1994; Nelson \& Bouton, 1997) emplearon un procedimiento de inhibición y otro de discriminación del rasgo negativo con el propósito de evaluar si las asociaciones inhibitorias son dependientes de contexto (cf. renovación). Los resultados mostraron que las asociaciones inhibitorias puras se transfieren correctamente entre contextos, mientras que las asociaciones inhibitorias establecidas con procedimientos de discriminación del rasgo negativo son específicas de contexto. Por tanto, estos autores sugirieron que las asociaciones establecidas durante extinción son específicas de este contexto no por su carácter inhibitorio, sino por ser la segunda asociación que establece el EC. Consistentemente, Nelson (2002) demostró que las asociaciones excitatorias también muestran dependencia contextual siempre y cuando se adquieran después de la adquisición de una asociación inhibitoria (i.e. sean la segunda información aprendida).

El estudio de los fenómenos de recuperación de respuestas refleja el interés por determinar los factores que modulan la extinción y la reaparición de respuestas, así como los mecanismos asociativos involucrados en estos fenómenos. Por tanto, no es de extrañar que se hayan propuesto diferentes explicaciones para cada uno de ellos. Sin embargo, el modelo de recuperación de información desarrollado por Bouton $(1997 ; 1991)$ ha recibido considerable atención, principalmente porque propone que la recuperación y el restablecimiento son variantes de la renovación contextual.

\section{MODELO DE RECUPERACIÓN DE INFORMACIÓN}

El modelo de recuperación de información asume que la memoria está conformada por nodos o unidades, que representan los eventos del ambiente. Estos nodos o unidades establecen asociaciones entre sí, a través de los mecanismos de aprendizaje asociativo. De acuerdo con el modelo de recuperación de información, la renovación contextual se explica considerando 
que durante la adquisición se establece una asociación entre la representación del EC y el EI, así cuando se presenta el EC el nodo que lo representa se activa y a través de la conexión establecida previamente, se excita el nodo del El, provocando así la emisión de la RC. Al finalizar la extinción, la asociación establecida durante la adquisición permanece intacta, mientras se establece una nueva asociación inhibitoria entre las representaciones del EC y el El. Como resultado de este proceso el significado del EC se hace ambiguo, es decir, tiene dos diferentes conexiones con el mismo El, una excitatoria y otra inhibitoria. De acuerdo con Bouton (1993, 1994), la activación de la asociación inhibitoria está modulada por el contexto donde se presenta el EC. Así, el contexto elimina la ambigüedad del EC seleccionando uno de los significados. Específicamente, el contexto excita un nodo intermedio que funciona como un puerto lógico AND, por tanto, cuando la representación del EC y del contexto se activan simultáneamente, la asociación inhibitoria suprime la activación del nodo del El por lo que se observa una ejecución similar a la extinción. Mientras que si la representación del contexto no se activa simultáneamente con la representación del EC, se activará la asociación excitatoria y se observará la renovación de la respuesta condicionada (i.e. una ejecución similar a la de adquisición). En conclusión, el principal supuesto del modelo es que para observar renovación la fase de prueba debe realizarse en un contexto diferente al empleado en la fase de extinción. Así, este modelo asume que el contexto no entra en asociación directa con la consecuencia, de hecho asume que el contexto afecta el significado del EC hasta la fase de extinción, cuando su significado se hace ambiguo.

\section{El modelo de recuperación de información y el restablecimiento}

Con relación al fenómeno de restablecimiento o reinstauración, el modelo de recuperación de información asume que la presentación no señalada del El provoca el condicionamiento del contexto. Por lo que la presentación del EC hace que el contexto de prueba se perciba como diferente del contexto de extinción, produciendo así la recuperación de la respuesta (Bouton y Ricker, 1994). Consistentemente, si la prueba ante el EC se conduce en un contexto diferente del utilizado para reexponer a los sujetos al El no se observa restablecimiento. Por ejemplo, Bouton y Bolles (1979b) mostraron que si se presenta el EC en un contexto que no ha sido apareado con el El no se puede observar el reestablecimiento de la respuesta. Si se toman en conjunto los hallazgos en renovación y reestablecimiento puede concluirse que la ejecución en la fase de prueba depende fundamentalmente de cuál de las dos representaciones (adquisición o extinción) se encuentra activa. 
El modelo de recuperación de información y la recuperación espontánea La explicación del modelo de recuperación de información sobre la recuperación espontánea es que el paso del tiempo en sí mismo puede funcionar como señal contextual. Al igual que una respuesta extinguida puede renovarse cuando el condicionamiento, la extinción y la prueba se realizan en contextos diferentes (A,B,C); la renovación contextual puede ocurrir con t1, t2, t3. Es decir, la recuperación espontánea se considera un fenómeno equivalente a la renovación $A B C$, por lo que en los procedimientos de recuperación espontánea el EC se prueba en un contexto temporalmente nuevo (Brooks \& Bouton, 1993; 1994).

La similitud en los resultados obtenidos con procedimientos de restablecimiento, renovación y recuperación espontánea sugiere que estos fenómenos pueden ser producto del mismo mecanismo: una falla en la recuperación de la extinción fuera del contexto de extinción (Bouton \& Nelson, 1998). Asimismo, esta evidencia sugiere que la extinción no lleva al desaprendizaje y que la ejecución después de la extinción depende del contexto en el que se presenta el EC. A pesar de que el modelo de recuperación de información predice con éxito los hallazgos descritos sobre renovación, restablecimiento y recuperación espontánea, a la fecha existen evidencia inconsistente con las predicciones de este modelo, la cual se describe a continuación.

\section{Evaluación del modelo de recuperación de información}

El modelo de recuperación de información predice renovación contextual en los diseños $A B A, A B C$ y $A A B$, ya que en todos ellos la prueba se conduce en un contexto diferente del empleado en extinción. Asimismo, el modelo predice que la magnitud de la renovación contextual será similar en los tres diseños. Sin embargo, varios estudios muestran diferencias en la magnitud de la renovación (Bouton \& Swartzentruber, 1989; Harris, Jones, Bailey \& Westbrook, 2000; Thomas, Larsen \& Ayres, 2003). Por ejemplo, Thomas et al. (2003) diseñaron un experimento con el objetivo de comparar la magnitud de la renovación en los diseños $A B A, A B C$ y $A A B$ empleando un procedimiento de supresión condicionada. En este experimento se emplearon tres contextos con distintas cualidades olfativas y de ubicación. Antes de iniciar el experimento, se pre-expuso a los grupos a los contextos y se les entrenó a presionar una palanca bajo un programa de intervalo variable $60 \mathrm{~s}$. En la fase de adquisición los sujetos recibieron emparejamientos entre el apagón de la luz general y una descarga eléctrica. Posteriormente, en la fase de extinción, se presentó para todos los grupos el apagón de luz en ausencia de la descarga. El grupo $A A B$ recibió esta fase en el mismo contexto de adquisición, mientras los grupos $A B A$ y $A B C$ la recibieron en un segundo contexto, denominado $B$. Finalmente, durante la fase de prueba se continúo la extinción, el grupo $A A B$ recibió esta fase en un contexto nuevo, denominado $B$, el grupo 
$A B C$ recibió esta fase en un tercer contexto, denominado $C$, y para el grupo ABA esta fase se condujo en el contexto de adquisición. Los resultados mostraron una recuperación de la respuesta de supresión en los tres grupos; sin embargo, la magnitud del efecto fue menor para el grupo AAB.

El hallazgo de que la renovación $A A B$ es de menor magnitud que la renovación $A B A$ y $A B C$, así como su sensibilidad a algunas manipulaciones (e.g. pre-exposición al contexto, número de ensayos de extinción) ha llevado a algunos autores a suponer que en este tipo de renovación podrían estar implicados mecanismos adicionales a los propuestos por el modelo de recuperación de información (Rosas, García-Gutiérrez \& Callejas-Aguilera, 2007; Sánchez-Carrasco, Bernal \& Nieto, 2009; Tamai \& Nakajima, 2000). Por ejemplo, Tamai y Nakajima (2000) contrastaron la renovación ABA y AAB después 18 ó 28 días de extinción. En la primera fase, se emparejó la presentación de un tono con una descarga en el contexto A. Posteriormente, se presentó el tono en extinción. Los sujetos de los grupos ABA-18 y ABA-28 recibieron esta fase en el contexto $B$, mientras los sujetos de los grupos AAB-18 y AAB-28 la recibieron en el contexto $A$. Asimismo, los grupos recibieron 18 ó 28 sesiones de extinción según su denominación. Finalmente, en la fase de prueba los grupos $A B A-18$ y $A B A-28$ regresaron al contexto $A$, mientras los grupos $A A B$ recibieron la prueba en el contexto $B$. Los resultados mostraron renovación de la respuesta de supresión en los grupos $A B A-18$ y ABA-28, mientras que sólo se observó renovación en el grupo $A A B-18$. Así, es posible concluir que la renovación $A B A$ no se ve afectada por el número de sesiones de extinción, mientras la renovación $A A B$ sí.

En conclusión, se ha observado la renovación $A B A, A B C$ y $A A B$ (Bouton, 2002) y en su mayoría los datos reportados en la literatura son consistentes con el modelo de recuperación de información. Sin embargo, la evidencia contradictoria con las predicciones del modelo puede resumirse de la siguiente manera: (1) Existen diferencias en la magnitud de la renovación $A B A, A B C$ y AAB (Thomas et al., 2003), (2) si se conduce un gran número de sesiones de extinción la renovación se atenúa (Denniston, Chang, y Miller, 2003) y (3) la evidencia sugiere que la renovación $A A B$ está controlada por factores diferentes a la renovación ABA y ABC (Rosas, et al., 2007; Sánchez-Carrasco, et al., 2009; Tamai \& Nakajima, 2000).

Desarrollo posterior del modelo de recuperación de información Recientemente, Rosas, Callejas-Aguilera, Ramos-Álvarez y Fernández-Abad (2006) han desarrollado un modelo que extiende el alcance de la propuesta realizada por Bouton y lo han denominado teoría atencional del procesamiento contextual. Esta teoría explica por qué la información adquirida en primer lugar es menos dependiente del contexto, mientras que la información adquirida en segundo lugar muestra mayor dependencia contextual. Asimismo, 
puede explicar por qué la recuperación de información es dependiente del contexto sin importar si la asociación establecida es inhibitoria o excitatoria, o si la información se adquirió en primer o segundo lugar.

La teoría atencional del procesamiento contextual comparte los siguientes supuestos con el modelo de recuperación de información: (1) las asociaciones establecidas durante el condicionamiento pueden interferirse mutuamente y (2) el contexto puede ser externo, interno, temporal o asociativo. Adicionalmente, Rosas y colaboradores sugieren que la dependencia contextual está determinada por la cantidad de atención que los sujetos animales y los participantes humanos prestan al contexto. Por tanto, la principal diferencia entre estos modelos está en el énfasis que se da al mecanismo mediante el cual la información se vuelve dependiente del contexto. Mientras que el modelo de recuperación de la información sugiere que la ambigüedad del EC provoca que las asociaciones sean dependientes del contexto, la teoría de Rosas et al. (2006) enfatiza que una vez que los sujetos atienden al contexto, la información adquirida posteriormente será dependiente de contexto.

Específicamente, la teoría atencional del procesamiento contextual propone cuatro factores que incrementan la atención de los sujetos al contexto: (1) la experiencia con el contexto, (2) las instrucciones, en preparaciones que utilizan participantes humanos, (3) el valor informativo del contexto o la presentación de información ambigua y (4) la saliencia del contexto. Por tanto, una vez que se presenta alguno de estos factores la información que se adquiera posteriormente mostrará dependencia contextual. Hasta ahora, existe alguna evidencia que sustenta las predicciones de la teoría atencional (Rosas \& Callejas-Aguilera, 2006; Rosas, García-Gutiérrez \& CallejasAguilera, 2006) sin embargo, es necesario determinar si todos los factores propuestos por Rosas y colaboradores incrementan la atención al contexto $y$, por tanto, favorecen la dependencia contextual de la información adquirida posteriormente.

En particular, existe evidencia de que la ambigüedad incrementa la atención al contexto. Rosas, García-Gutiérrez et al. (2006) entrenaron a participantes humanos en una preparación de juicios predictivos. Inicialmente se emparejó una señal $(X)$ con una consecuencia $(C 1)$. Posteriormente, $X$ se emparejó con otra consecuencia $(C 2)$, mientras otra señal $(Y)$ se emparejó con la C1. Los resultados de los juicios predictivos mostraron que la asociación Y-C1 era dependiente de contexto. Adicionalmente, una tercera señal $(Z)$ mostró dependencia contextual a pesar de ser entrenada fuera del contexto de interferencia. Estos resultados sustentan la propuesta de que una vez que los participantes atienden al contexto, la información que se adquiere posteriormente también mostrará dependencia contextual. 


\section{CONCLUSIONES}

En conclusión, la evidencia revisada anteriormente indica que la extinción de una respuesta condicionada pavloviana o instrumentalmente no implica su desaparición permanente. Por el contrario, se han identificado diversas condiciones que permiten la reaparición de las respuestas minutos u horas después de su extinción. Bouton $(1993,1994)$ propuso un modelo teórico que ha guiado mucha de la investigación y discusión teórica de estos fenómenos. Sin embargo, a pesar de su éxito explicativo, se han empezado a acumular reportes que hacen necesario considerar la evidencia que contradice el modelo de recuperación de información y desarrollar nuevas predicciones que permitan explicar el mecanismo que subyace a la extinción, la dependencia contextual y la recuperación de información. Asimismo, es relevante determinar si los fenómenos de recuperación de información comparten mecanismos asociativos comunes o diferentes, así como si estos fenómenos interactúan entre sí produciendo una mayor recuperación en la respuesta.

En este contexto, es de particular interés en el desarrollo de cualquier modelo dentro de esta área considerar las afinidades entre la renovación $A B A$ y $A B C$. En estos diseños el cambio de contexto correlaciona con el cambio de significado del EC o la respuesta instrumental (Pineño \& Miller, 2004), mientras que la renovación $A A B$ refleja simplemente el efecto de extraer al sujeto del contexto de extinción. Consistentemente, varios autores han sugerido que es necesario identificar los mecanismos adicionales que subyacen a la renovación $A B A$ y $A B C$ (Rosas, Callejas-Aguilera et al., 2006). Asimismo, recientemente se ha considerado la posibilidad de que durante la adquisición los sujetos presten atención al contexto y, por tanto, la información adquirida en esta fase muestre también dependencia contextual (Pineño \& Miller, 2004; Tamai \& Nakajima, 2000). Por consiguiente, es posible suponer que la renovación $A A B$ y $A B C$ muestran el mecanismo de configurador de ocasión propuesto por Bouton (1993), mientras que en la renovación ABA se presenta adicionalmente una señal asociada con el contexto de adquisición (Tamai \& Nakajima, 2000).

\section{REFERENCIAS}

Ahlers, S. T. \& Richardson, R. (1985). Administration of dexamethasone prior to training blocks ACTCH-induced recovery of an extinguished avoidance response. Behavioural Neuroscience, 99, 760-764.

Baker, A. G., Steinwald, H. \& Bouton, M. E. (1991). Contextual conditioning and reinstatement of extinguished instrumental responding. The Quaterly Journal of Experimental Psychology, 43B, 199-218. 
Bouton, M. E. (1993). Context, time and memory retrieval in the interference paradigms of pavlovian learning. Psychological Bulletin, 114, 80-99.

Bouton, M. E. (1994). Conditioning, remembering and forgetting. Journal of Experimental Psychology: Animal Behavior Processes, 20, 219-231.

Bouton, M. E. \& Bolles, R. C. (1979a). Contextual control of extinction of conditioned fear. Learning and Motivation, 10, 445-466.

Bouton, M. E. \& Bolles, R. C. (1979b). Role of conditioned contextual stimuli in reinstatement of extinguished fear. Journal of Experimental Psychology: Animal Behavior Processes, 5, 368-378.

Bouton, M. E., Kenney, F. A. \& Rosengard, C. (1990). State-dependent fear extinction with two benzodiazepine tranquilizers. Behavioural Neuroscience, 104, 44-55.

Bouton, M. E. \& Nelson, J. B. (1994). Context-specificity of target versus feature inhibition in a feature negative discrimination. Journal of Experimental Psychology: Animal Behavior Processes, 20, 51-65.

Bouton, M. E. \& Peck, C. A. (1989). Context effects on conditioning, extinction and reinstatement in an appetitive conditioning preparation. Animal Learning \& Behavior, 17(2), 188-198.

Bouton, M. E. \& Ricker, S. T. (1994). Renewal of extinguished responding in a second context. Animal Learning \& Behavior, 22, 317-324.

Bouton, M. E. \& Swartzentruber, D. (1986). Analysis of the associative and occasionsetting properties of contexts participating in a Pavlovian discrimination. Journal of Experimental Psychology: Animal Behavior Processes, 12, 333-350.

Bouton, M. E. \& Swartzentruber, D. (1989). Slow reacquisition following extinction: Context, encoding and retrieval mechanisms. Journal of Experimental Psychology: Animal Behavior Processes, 15, 43-53.

Brooks, D. C. \& Bouton, M. E. (1993). A retrieval cue for extinction attenuates spontaneous recovery. Journal of Experimental Psychology: Animal Behavior Processes, 19, 77-89.

Brooks, D. C. \& Bouton, M. E. (1994). A retrieval cue for exctinction attenuates response recovery (renewal) caused by a return to the conditioning context. Journal of Experimental Psychology: Animal Behavior Processes, 20, 366-379.

Colwill, R. M. (1993). An associative analysis of intrumental learning. Current Directions in Psychological Science, 2, 111-116.

Colwill, R. M. \& Rescorla, R. A. (1988). Associations between the discriminative stimulus and the reinforcer in instrumental learning. Journal of Experimental Psychology: Animal Behavior Processes, 14, 155-164.

Delamater, A. R. (1997). Selective reinstatemente of stimulus-outcome associations. Animal Learning \& Behavior, 25, 400-412.

Denniston, J. C., Chang, R. C. \& Miller, R. R. (2003). Massive extinction treatment attenuates the renewal effect. Learning and Motivation, 34, 68-86.

Dickinson, A. (1980). Contemporary animal learning theory. Cambridge, Massashusets: Cambridge University Press.

Doughty, A., Reed, P. \& Lattal, K. A. (2004). Differential reinstatement predicted by preextinction response rate. Psychonomic Bulletin and Review, 11, 1118-1123.

Ellson, D. G. (1938). Quantitative studies of the interaction of simple habits: I Recovery from specific and generalized effects of extinction. Journal of Experimental Psychology, 23, 339-358. 
Estes, W. K. (1950). Toward a statistical theory of learning. Psychological Review, 57, 94-107.

Franks, G. J. \& Lattal, K. A. (1976). Antecedent reinforcement schedule training and operant response reinstatement in rats. Animal Learning \& Behavior, 4, 374-378.

Gámez, A. M. \& Rosas, J. M. (2005). Transfer of stimulus control across instrumental responses is attenuated by extinction in human instrumental conditioning. International Journal of Psychology and Psychological Therapy, 5, 207-222.

Hall, G. (2002). Associative structures in Pavlovian and instrumental conditioning. In C. R. Gallistel (Ed.), Stevens' handbook of experimental psychology, (vol. 3, pp. 1-45). New York: John Wiley y Sons.

Harris, J. A., Jones, L., Bailey, G. K. \& Westbrook, R. F. (2000). Contextual control over conditioned responding in an extinction paradigm. Journal of Experimental Psychology: Animal Behavior Processes, 26, 174-185.

Konorski, J. (1948). Conditied reflexes and neuronal organization. Londres: Cambridge University Press.

Nakajima, S., Tanaka, S., Urushihara, K. \& Imada, H. (2000). Renewal of extinguished lever-press responses upon return to the training context. Learning and Motivation, 31, 416-431.

Nelson, J. B. (2002). Context specificity of excitation and inhibition in ambiguous stimuli. Learning and Motivation, 28, 56-84.

Nelson, J. B. \& Bouton, M. E. (1997). The effects of a context switch following serial and simultaneous feature-negative discriminations. Learning and Motivation, 28, 56-84.

Ostlund, S. B. \& Balleine, B. W. (2007). Selective reinstatement of instrumental performance depends on the discriminative stimulus properties of the mediating outcome. Learning \& Behavior, 35, 43-52.

Paredes-Olay, C. \& Rosas, J. M. (1999). Within-subjects extinction and renewal in predictive judgments. Psicológica, 20, 195-210.

Pavlov, I. P. (1927). Conditioned reflex. Londres: Oxford University Press.

Pineño, O. \& Miller, R. R. (2004). Signaling a change in cue-outcome relations in human associative learning. Learning \& Behavior, 32, 360-375.

Reid, R. L. (1958). The role of the reinforcer as a stimulus. British Journal of Psychology, 49, 202-209.

Rescorla, R. A. (1979). Conditioned inhibition and excitation. En A. Dickinson \& R. A. Boakes (Eds.), Mechanisms of learning and memory: A memorial volume to Jerzy Konorski. Hillsdale, NJ: Erlbaum.

Rescorla, R. A. (1992). Associations between an instrumental discriminative stimulus and multiple outcomes. Journal of Experimental Psychology: Animal Behavior Processes, 18, 95-104.

Rescorla, R. A. (1993). Preservation of response-outcome associations through extinction. Animal Learning \& Behavior, 21, 238-245.

Rescorla, R. A. (1996a). Preservation of Pavlovian associations through extinction. The Quaterly Journal of Experimental Psychology, 49B, 245-258.

Rescorla, R. A. (1996b). Response-outcome associations remain funcitional through interference treatments. Animal Learning \& Behavior, 24, 450-458. 
Rescorla, R. A. (2001). Are associative changes in acquisition and extinction negatively accelerated? Journal of Experimental Psychology: Animal Behavior Processes, 27, 307-315.

Rescorla, R. A. \& Cunningham, C. L. (1977). The erasure of reinstated fear. Animal Learning \& Behavior, 5, 386-394.

Rescorla, R. A. \& Heth, C. D. (1975). Reinstatement of fear to an extinguished conditioned stimulus. Journal of Experimental Psychology: Animal Behavior Processes, 1, 88-96.

Rescorla, R. A. \& Skucy, J. C. (1969). The effect of response-independent reinforcers during extinction. Journal of Comparative \& Physiological Psychology, 67, 381389.

Rescorla, R. A. \& Wagner, A. R. (1972). A theory of Pavlovian conditioning: Variations in the efectivness of reinforcement and nonreinforcement. En A. H. Black y W. F. Prokasy (Eds.), Classical Conditioning II: Current research and theory (pp. 6499). Nueva York: Appleton-Century-Crofts.

Robbins, S. J. (1990). Mechanisms underlying spontaneous recovery in autoshaping. Journal of Experimental Psychology: Animal Behavior Processes, 16, 235-249.

Rosas, J. M. \& Bouton, M. E. (1997). Renewal of a conditioned taste aversion upon return to the conditioning context after extinction in another one. Learning and Motivation, 28, 216-229.

Rosas, J. M. \& Callejas-Aguilera, J. E. (2006). Context switch effects on acquisition and extinction in human predictive learning. Journal of Experimental Psychology: Learning, Memory, and Cognition, 32, 461-474.

Rosas, J. M., Callejas-Aguilera, J. E., Ramos-Álvarez, M. M. \& Férnandez-Abad, M. J. (2006). Revision of retrieval theory of forgetting: What does make information context-specific? International Journal of Psychology and Psychological Therapy, 6, 147-166.

Rosas, J. M., García-Gutiérrez, A. \& Callejas-Aguilera, J. E. (2006). Effects of context change upon retrieval of first and second-learned information in human predictive learning. Psicologica, 27, 35-56.

Rosas, J. M., García-Gutiérrez, A. \& Callejas-Aguilera, J. E. (2007). AAB and ABA renewal as a function of the number of extinction trials in conditioned taste aversion. Psicologica, 28, 129-150.

Sánchez-Carrasco, L., Bernal, R. \& Nieto, J. (2009). ABA, ABC, and AAB renewal in an Instrumental procedure. Manuscrito enviado a publicación.

Skinner, B. F. (1950). Are theories of learning necessary? Psychological Review, 57, 193-216.

Tamai, N. \& Nakajima, S. (2000). Renewal of formerly conditioned fear in rats after extensive extinction training. International Journal of Comparative Psychology, $13,137-147$.

Thomas, B. L., Larsen, N. \& Ayres, J. J. B. (2003). Role of context similarity in ABA, $A B C$ and $A A B$ renewal paradigms: Implications for thoeries of renewal and for treating human phobias. Learning and Motivation, 34, 410-436.

Uhl, C. N. (1973). Eliminating behavior with omission and extinction after varying amounts of training. Animal Learning \& Behavior, 1, 237-240. 